\title{
Docetaxel chemotherapy for metastatic hormone refractory prostate cancer as first-line palliative chemotherapy and subsequent re-treatment: Birmingham experience
}

\author{
JAWAHER ANSARI ${ }^{1}$, SYED ANWER HUSSAIN $^{1}$, ANJALI ZARKAR ${ }^{1}$, \\ JACOB S. TANGUAY ${ }^{1}$, JULIE BLISS ${ }^{2}$ and JOHN GLAHOLM ${ }^{1}$ \\ ${ }^{1}$ Queen Elizabeth Hospital, Vincent Drive, Birmingham B15 2TH; \\ ${ }^{2}$ Good Hope Hospital, Sutton Coldfield, Birmingham B75 7RR, UK
}

Received April 17, 2008; Accepted June 27, 2008

DOI: 10.3892/or_00000088

\begin{abstract}
Three-weekly docetaxel chemotherapy with prednisolone is now considered standard of care for patients with metastatic hormone refractory prostate cancer (MHRPC). This study reports the efficacy and toxicity of first-line docetaxel chemotherapy followed subsequently by re-treatment on biochemical disease progression (BDP). Forty-two patients with MHRPC were treated with three-weekly docetaxel chemotherapy $75 \mathrm{mg} / \mathrm{m}^{2}$ and $10 \mathrm{mg}$ of prednisolone daily. Median age 73 years (range 58-87) and median initial PSA $182 \mathrm{ng} / \mathrm{ml}$ (range 19.9-1500). Of these patients, 10 were re-treated with the same regimen (second-line chemotherapy) on BDP. A further 3 out of these 10 patients received 2nd re-treatment (third-line chemotherapy) with docetaxel chemotherapy on BDP. Fifty-four percent of patients responded to first-line docetaxel chemotherapy and all re-treated patients responded again with a PSA reduction $>50 \%$. Median treatment-free interval prior to second and third-line chemotherapy was 24 and 26 weeks, respectively. Grade 3 or 4 neutropenia occurred in 2.5, 7 and $12 \%$ of the total number of cycles in patients receiving first-, second- and third-line docetaxel chemotherapy, respectively. Median survival was 13 months (range 3-35) and one-year overall survival $52 \%$. This is the first report of three-weekly docetaxel chemotherapy re-treatment in patients with MHRPC and demonstrates that patients who initially respond to docetaxel chemotherapy maintain their sensitivity to subsequent retreatment without a significant rise in haematological toxicity.
\end{abstract}

Correspondence to: Dr Jawaher Ansari, University Hospital Birmingham NHS Trust, Metchley Drive, Birmingham B15 2TH, UK E-mail: drjawaher@yahoo.com

Key words: prostate cancer, hormone refractory disease, chemotherapy, docetaxel, second-line chemotherapy, third-line chemotherapy, docetaxel re-treatment

\section{Introduction}

Prostate Cancer is the most common cancer in men in the UK, accounting for one in five of all new male cancers. Incidence rates increase steeply with age, with $>65 \%$ cases occurring in men over the age of 70 years (1). Androgen ablation, with either Leutinizing Hormone Releasing Hormone (LHRH) agonists or less commonly with surgical orchidectomy, remains the primary modality of treatment for metastatic prostate cancer. Androgen ablation is highly effective, providing disease control in nearly $80 \%$ of patients. Despite an initial good response, nearly all patients will become refractory to LHRH analogues after a median duration of 18-24 months, requiring alternative treatment options. Hormone Refractory Prostate Cancer (HRPC) is defined either on the basis of rising prostate-specific antigen (PSA), or with evidence of clinical or radiological disease progression despite castrate levels of testosterone. Treatment options for metastatic Hormone Refractory Prostate Cancer (MHRPC) include second-line hormone manipulations, palliative chemotherapy, bisphosphonates, radio-isotopes, or best supportive care. The role of continued androgen suppression following diagnosis of MHRPC is controversial, although there is evidence suggestive of a moderate survival benefit for this approach (2). Options for hormonal manipulations include the addition of anti-androgens, followed by withdrawal, corticosteroids or oestrogen. Response to second-line hormonal therapy is short-lived and does not impact on overall survival. 'Antiandrogen withdrawal response' has been documented in up to $25 \%$ of patients with HRPC. Response rates between 5 and $30 \%$ have been documented using anti-androgens or glucocorticoids as second-line hormonal treatments for HRPC (3).

Historically, prostate cancer has been considered to be a chemo-resistant disease with earlier trials showing disappointing response rates $(<20 \%)$. However, in 1996 Tannock et al published their randomised study revealing the first evidence of palliative benefit of mitoxantrone and prednisolone over prednisolone alone in patients with symptomatic MHRPC (4). Subsequent studies confirmed the same results but failed to a show a survival benefit for chemotherapy, until in 2004, two landmark studies (TAX-327 
and SWOG 99-16) provided the first evidence of survival benefit with docetaxel-based chemotherapy for patients with MHRPC. In the TAX-327 study, 1006 men with MHRPC received prednisolone $5 \mathrm{mg}$ twice daily and were randomised to receive mitoxantrone $12 \mathrm{mg} / \mathrm{m}^{2}$ every three weeks, docetaxel $75 \mathrm{mg} / \mathrm{m}^{2}$ every three weeks, or docetaxel $30 \mathrm{mg} / \mathrm{m}^{2}$ weekly for five out of every six weeks. Results from this study confirmed superiority of the three-weekly docetaxel regimen over mitoxantrone with a significant improvement in median survival (18.9 vs. 16.5 months; $\mathrm{P}=0.009)$, PSA response (45 vs. $32 \% ; \mathrm{P}<0.001)$ and pain response (35 vs. $22 \%$; $\mathrm{P}=0.01$ ) (5). Results from the SWOG 99-16 study showed that patients treated with a combination of docetaxel and estramustine had a significant improvement in median overall survival (17.5 vs. 15.6 months; $\mathrm{P}=0.02)$ and PSA response (50 vs. $27 \%$; $\mathrm{P}<0.001$ ) when compared to mitoxantrone and prednisolone (6). Based on the results of these studies, the National Institute for Health and Clinical Excellence (NICE) committee approved ten cycles of threeweekly docetaxel chemotherapy $\left(75 \mathrm{mg}\right.$ per $\left.\mathrm{m}^{2}\right)$ in combination with prednisolone $10 \mathrm{mg}$ daily as standard of care for first-line chemotherapy in patients with MHRPC (7).

Despite the widespread use of docetaxel chemotherapy in patients with MHRPC, several questions remain unanswered, including a) the optimal timing of chemotherapy, b) the number of cycles needed, c) the options for second-line chemotherapy in responders who progress after a period of biochemical remission and finally d) the role of intermittent chemotherapy.

Currently, none of the available chemotherapeutic agents are licensed for second-line chemotherapy in patients with MHRPC. Chemotherapy options for docetaxel-refractory patients are extremely limited. Mitoxantrone, vinorelbine, carboplatin and etoposide, carboplatin and docetaxel combinations seem to be used widely outside a clinical trial setting, though the evidence from phase II studies indicates response rates $<20 \%(8-10)$. It is less clear how to manage patients who respond to docetaxel initially and then progress after a period of biochemical remission. In view of the lack of evidence for this category of patients, the NICE committee has not approved docetaxel re-treatment (7). However, due to the lack of treatment options for second-line chemotherapy, we have been routinely re-treating MHRPC patients who initially respond to docetaxel chemotherapy and subsequently progress after achieving a period of biochemical remission.

We have therefore reviewed our database of MHRPC patients receiving docetaxel chemotherapy to assess whether the efficacy and toxicity of treatment correlates with clinical trial reported data. We have also reviewed the feasibility and efficacy of re-treatment with 3-weekly docetaxel chemotherapy (75 $\mathrm{mg}$ per $\mathrm{m}^{2}$ ), as this has not been reported previously.

\section{Patients and methods}

Between August 2004 and September 2007, 42 patients with MHRPC were treated with docetaxel chemotherapy $75 \mathrm{mg} / \mathrm{m}^{2}$ intravenously on day 1 repeated every three weeks along with prednisolone $10 \mathrm{mg}$ daily. All patients who had received more than one cycle of docetaxel chemotherapy were included in the study. Androgen suppression with LHRH analogues was
Table I. Baseline patient characteristics.

\begin{tabular}{lc}
\hline Median age (yrs) & $73(58-87)$ \\
Median PSA (ng/ml) & 182 \\
PSA >20 ng/ml (\%) & 98 \\
$>2$ Hormone manipulations (\%) & 95 \\
Gleason score (\%) & \\
$\leq 7$ & 19 \\
$8-10$ & 38 \\
Prior treatment (\%) & \\
Prostatectomy & 5 \\
Radiotherapy & 26 \\
Site of metastases (\%) & \\
Bone only & 74 \\
Visceral disease only & 7 \\
Bone and visceral & 19 \\
\hline
\end{tabular}

continued throughout the duration of chemotherapy. We retrospectively reviewed patient notes to assess baseline patient characteristics (Table I), PSA response, haematological toxicity, progression-free interval and overall survival. We aimed to deliver 10 cycles of three-weekly docetaxel chemotherapy along with prednisolone to all patients, unless they developed significant toxicity or evidence of clinical, radiological or biochemical disease progression during treatment. Due to the lack of alternative options for secondand third-line chemotherapy, patients who progressed after an initial biochemical response were offered re-treatment with docetaxel and prednisolone. Of the 42 patients, 10 were retreated with docetaxel $\left(75 \mathrm{mg}\right.$ per $\mathrm{m}^{2}$ intravenously on day 1 repeated every three weeks) and prednisolone (10 $\mathrm{mg}$ daily) as second-line chemotherapy (1st re-treatment) on biochemical disease progression. A further 3 of these 10 patients were re-treated with the same regimen as third-line chemotherapy (2nd re-treatment) on disease progression. We reviewed response rates, toxicities, progression-free interval and overall survival for first-line docetaxel chemotherapy and subsequent re-treatments with the three-weekly chemotherapy regimen. Data for overall survival was compiled from patient notes and the National Health Service strategic tracking service. Survival was defined from the start of first-line palliative chemotherapy to the date of death from any cause or censored at the date of last contact. Median overall survival was analysed by means of the Kaplan-Meier method.

Response criteria. The majority of patients have bone metastases only and this therefore makes it impractical to use Response Evaluation Criteria in Solid Tumours (RECIST) criteria for assessment of response. Demonstration of survival benefit is the gold standard for any treatment option for MHRPC. However, based on the outcome of the Consensus conference in 1999, PSA decline of $50 \%$ or more is considered to be a valid end-point when reporting response rates in patients with metastatic prostate cancer (11). More 
recently, re-analysis of data from the TAX-327 and SWOG 99-16 studies revealed that a PSA decline of $30 \%$ or more with treatment is the best surrogate marker for overall survival $(12,13)$. Based on these results we quantified PSA response into two groups; group 1 with PSA reduction of at least $50 \%$ and group 2 with a PSA reduction between 30 and $50 \%$ of the baseline serum PSA values. Patients with a PSA rise $>50 \%$ of the pre-treatment level were classed as refractory disease. Patients whose PSA response did not meet the above criteria were regarded as having stable disease. Biochemical response could not be assessed in patients whose PSA values were $>1500 \mathrm{ng} / \mathrm{ml}$. Haematological toxicity was evaluated by means of the Common Toxicity Criteria of the National Cancer Institute (version 3.0).

\section{Results}

First-line docetaxel chemotherapy. A total of 42 patients with MHRPC were treated with docetaxel chemotherapy along with prednisolone using the three-weekly regimen as detailed previously. Baseline characteristics of these patients are reported in Table I. The median age was 73 years and $40 \%$ of patients were aged at least 75 years. Forty-three percent of patients had metastatic disease at presentation and $57 \%$ were symptomatic prior to commencement of chemotherapy. The median PSA at commencement of chemotherapy was $182 \mathrm{ng} /$ $\mathrm{ml}$ (range 19.9->1500). Serum PSA measurements were checked at baseline and repeated at three weekly intervals during treatment. Thirty-one percent of patients completed the planned course of 10 cycles of chemotherapy and the median number of cycles delivered as part of first-line palliative chemotherapy was seven (range 2-10). Forty percent of patients had an excellent biochemical response, achieving a PSA decline $>50 \%$. Additionally, another $14 \%$ of patients had PSA reduction between 30 and $50 \%$ of the pre-treatment levels. Biochemical response could not be assessed in three patients as their baseline PSA readings were $>1500 \mathrm{ng} / \mathrm{ml}$ and remained at the same level during treatment. Sixteen percent of patients had stable PSA measurements, whereas another $23 \%$ had progressive disease during treatment. Twenty out of 25 symptomatic patients $(80 \%)$ reported an improvement in symptom control with chemotherapy. The majority of patients received the prescribed dose of docetaxel, with only $14 \%$ needing a dose reduction. Two hundred and ninety one cycles of chemotherapy were delivered and haematological toxicity is summarised in Fig. 2A. Four cycles were complicated by grade 3 neutropenia and three cycles by grade 4 neutropenia. Overall incidence of grade 3 or 4 neutropenia was relatively low, affecting $17 \%$ of patients $(n=7)$ and complicating only $2.5 \%$ of the total number of cycles. Only one patient required hospital admission for management of neutropenic sepsis with no treatment-related deaths. Grade 3 or 4 anaemia was observed in only $5 \%$ of patients, although 8 patients (19\%) required at least one unit of blood transfusion during treatment. There were no episodes of grade 3 or 4 thrombo-cytopenia. At the time of analysis, 22 out of 42 patients were alive at 12 months (52\%) and only 6 patients had follow-up $<12$ months. The median duration of survival was 13 months (range 3-35). A KaplanMeier survival curve for this group is shown in Fig. 1.

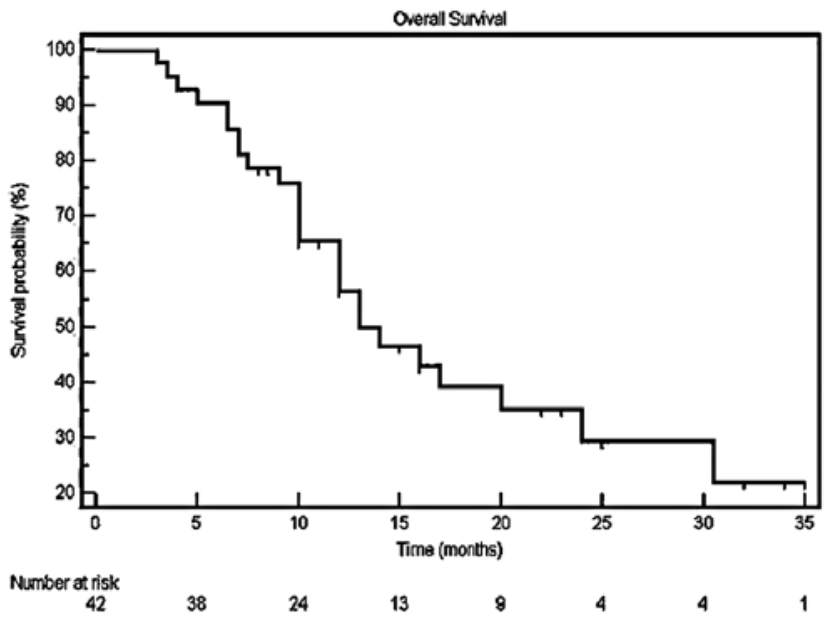

Figure 1. Kaplan-Meier plot of overall survival for the 43 patients with MHRPC.

Second-line chemotherapy (first re-treatment). Of the 42 patients, 10 were re-treated with docetaxel chemotherapy on biochemical disease progression (regimen as described previously). The median age of patients receiving second-line chemotherapy was 67 years (range 59-79). Fifty-nine cycles of docetaxel chemotherapy were delivered to 10 patients with a median of 6 cycles per patient (range 2-10). The median treatment-free interval prior to re-treatment was 24 weeks (range 14-41 weeks). Following first-line docetaxel chemotherapy treatment, 7 out of 10 patients had a good biochemical response (PSA reduction $>50 \%$ ), two patients had stable disease and one patient had un-recordable PSA $>1500 \mathrm{ng} / \mathrm{ml}$. On re-treatment with docetaxel chemotherapy, all seven patients who had responded initially maintained their intrinsic sensitivity to chemotherapy and responded again with a PSA reduction $>50 \%$. The remaining three patients had symptom improvement following re-treatment with docetaxel chemotherapy. Haematological toxicity is summarised in Fig. 2B. Out of 59 chemotherapy cycles, grade 3 neutropenia was observed in three cycles and grade 4 neutropenia was observed in one cycle. Grade 3 or 4 neutropenia was observed in $20 \%$ of patients and complicated $7 \%$ of the total number of cycles. Compared to first-line docetaxel chemotherapy, there was an increase in haematological toxicity with re-treatment, though this did not reach statistical significance $(\mathrm{P}=0.09)$. Only one patient required hospital admission for management of neutropenic sepsis with no treatment-related deaths. There were no episodes of grade 3 or 4 thrombocytopenia or anaemia.

Third-line chemotherapy (second re-treatment). Of the 7 patients who responded to first- and second-line docetaxel chemotherapy, three received further re-treatment with seventeen cycles of docetaxel as third-line chemotherapy on biochemical disease progression. The median treatment-free interval prior to third-line chemotherapy was 26 weeks (range 16-47). Having responded to first- and second-line docetaxel chemotherapy, all three patients maintained their sensitivity to docetaxel and responded to third-line chemotherapy with a PSA reduction $>50 \%$ of the baseline 
$\mathbf{A}$

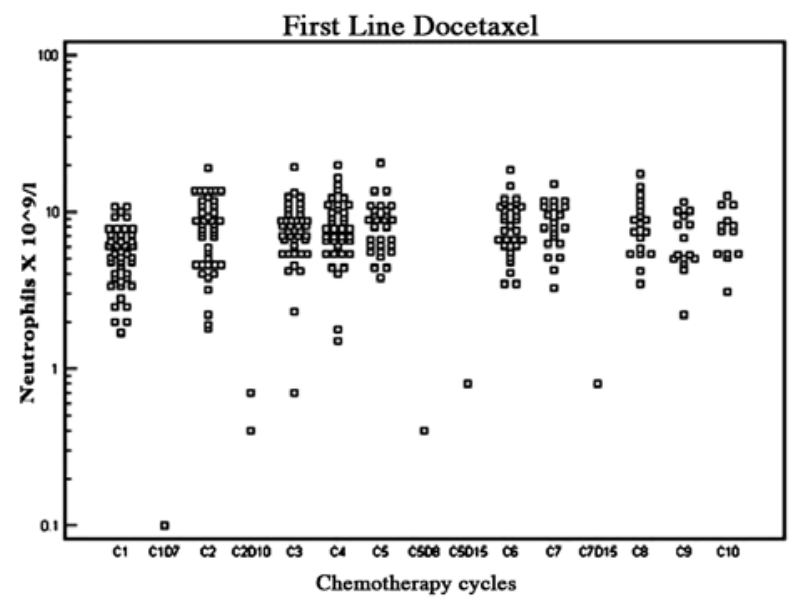

B

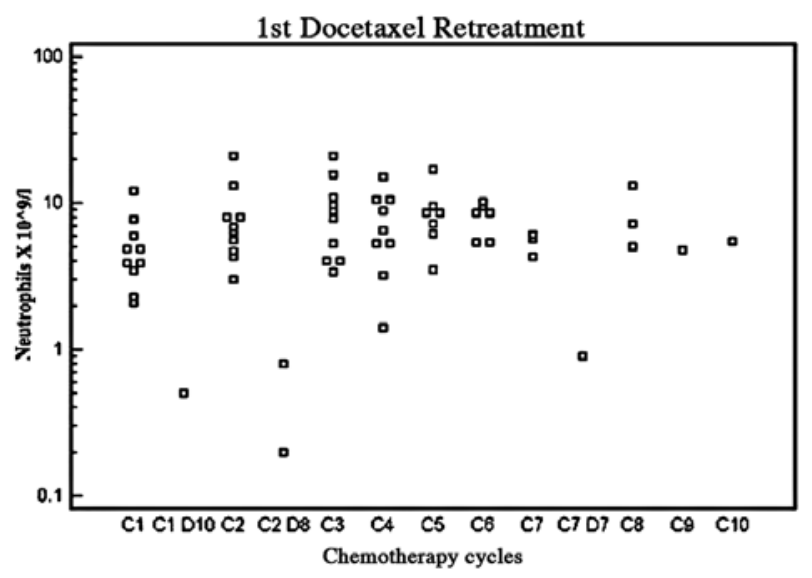

C

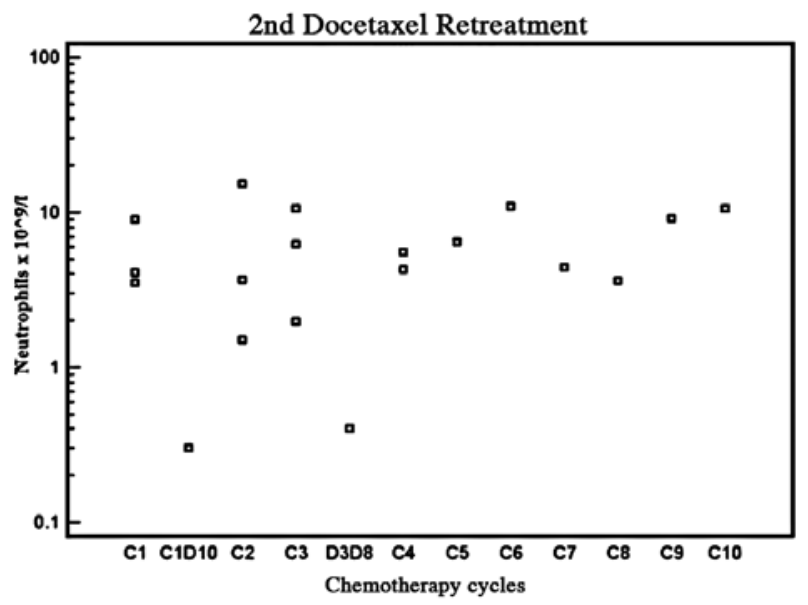

Figure 2. (A) Neutrophil counts with first-line docetaxel chemotherapy. (B) Neutrophil counts with second-line docetaxel chemotherapy. (C) Neutrophil counts with third-line docetaxel chemotherapy.

values (Fig. 3). Haematological toxicity is summarised in Fig. 2C. Grade 4 neutropenia was observed in two cycles and complicated $12 \%$ of the total number of cycles. Despite the increase in toxicity, there were no treatment-related deaths. There were no episodes of grade 3 or 4 thrombocytopenia or anaemia.

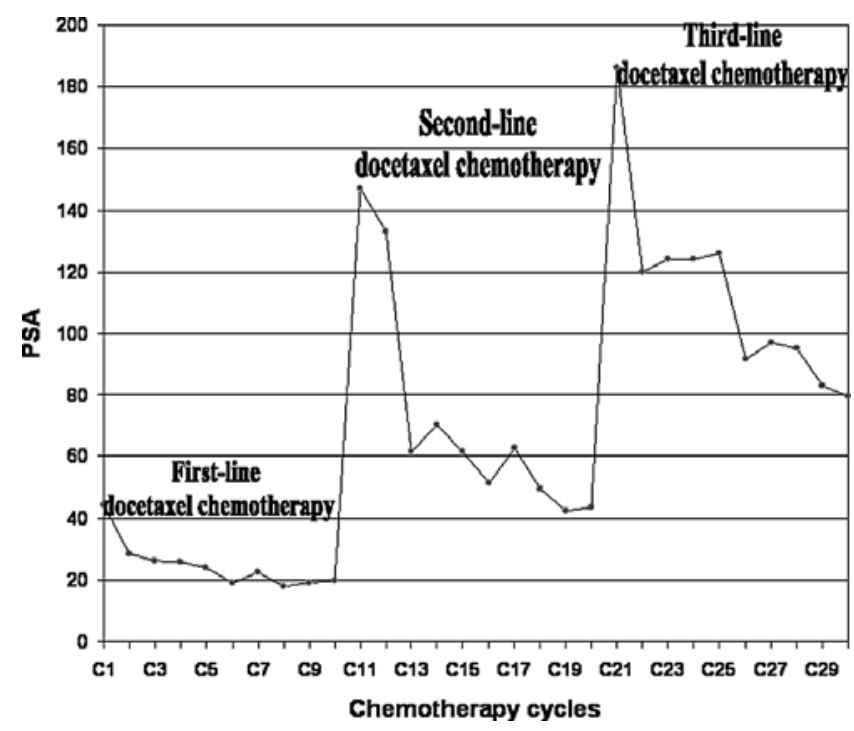

Figure 3. PSA response in a patient with MHRPC who received 30 cycles of three-weekly docetaxel chemotherapy (10 cycles each of first-, second- and third-line chemotherapy).

\section{Discussion}

Due to advanced radiological investigations and increased frequency of PSA monitoring, the diagnosis of disease progression is being made earlier in patients with MHRPC. A significant proportion of these patients will be candidates for second-line chemotherapy, having previously responded to docetaxel and prednisolone as first-line chemotherapy.

We performed a literature review looking for second-line chemotherapy options and evidence for docetaxel re-treatment in patients with MHRPC. Not surprisingly, there have been no previous reports of docetaxel re-treatment or intermittent administration using the three-weekly regimen in patients with MHRPC. Although, the NICE committee have recommended against re-treatment for this group of patients, there are no other suitable licensed treatment options assuming other hormonal options are exhausted prior to commencement of chemotherapy (7). Though the evidence for chemotherapy re-treatment in MHRPC is limited, there is sufficient evidence in other solid tumours and haematological malignancies that re-treatment with the same regimen following complete remission and subsequent relapse can offer response rates between 18 and $100 \%$ (median 51\%) (14). Activity of docetaxel chemotherapy following failure of first-line mitoxantrone in HRPC patients has been reported in several small phase II studies and retrospective series with response rates between 35 and $50 \%$ (15-17).

The role of intermittent versus continuous androgen suppression has been explored in at least three randomised controlled trials. Although, there was no difference in time to progression or overall survival it is interesting to note that the majority of patients achieved normal testosterone levels during the treatment-free period $(18,19)$. Similarly, intermittent administration of chemotherapy is an attractive proposition which may offer patients improved quality of life during the treatment-free periods. Beer et al prospectively tested intermittent weekly docetaxel and calcitriol in eight patients with HRPC, achieving a significant treatment-free interval 
(nearly 20 weeks) with this approach (20). ASCENT, a phase II randomised study compared docetaxel $\left(36 \mathrm{mg} / \mathrm{m}^{2}\right)$ administered weekly intravenously for 3 out of 4 weeks in combination with either calcitriol $(45 \mu \mathrm{g})$ or placebo taken weekly in patients with MHRPC. Intermittent chemotherapy administration was allowed for patients achieving a significant biochemical response. Interestingly, of the 45 patients who received intermittent chemotherapy, $45.5 \%$ showed a biochemical response (PSA reduction $>50 \%$ ) on being rechallenged with the same regimen after a median first treatment-free interval of 18 weeks (21). However, results from these studies are limited by small sample size (21) and use of non-standard chemotherapy regimen (21) (Petrylak DP, et al, 3rd annual ASCO Prostate Cancer Symposium, 145, 2007).

The role of second-line mitoxantrone chemotherapy has been studied by Michels et al in a population-based retrospective study comparing the effectiveness of sequencing docetaxel and mitoxantrone chemotherapy as first- and second-line treatment for patients with HRPC (17). The study included 68 patients with HRPC who had received docetaxel (either weekly or three-weekly regimen) and mitoxantrone chemotherapy in either sequence. Compared to mitoxantrone, second-line treatment with docetaxel showed a higher PSA response (38 vs. 12\%), with no difference in overall survival. Despite its limitations, this study provided some evidence that both docetaxel and mitoxantrone have activity as second-line chemotherapy drugs in patients with MHRPC. Novel approaches for management of MHRPC are being investigated, although the majority of these agents are still in early clinical studies. Ixabepilone, an epothilone B analogue has shown modest activity as second-line treatment for docetaxelrefractory HRPC patients with PSA response rates $<20 \%$ (8). Satraplatin, a third-generation orally administered platinum analogue has recently shown activity as second-line chemotherapy in patients with HRPC. SPARC, an international, multi-centre, phase III double-blind placebo controlled trial, randomised 950 patients with HRPC to satraplatin and prednisone or placebo and prednisone after failure of one prior chemotherapy regimen. Patients in the satraplatin arm had a $13 \%$ improvement in median PFS (11 vs. 9.7 weeks), improvement in pain response (24 vs. 14\%) and PSA response (35 vs. 12\%). However, it is interesting to note that the definition of disease progression in this trial was a composite unconventional end-point comprising radiographic and symptomatic progression, but did not include PSA progression. Based on the results of this study, satraplatin has been submitted for accelerated FDA approval in the United States as second-line chemotherapy for patients with MHRPC. Our study compares favourably to the above studies and shows encouraging efficacy. It was well tolerated when patients were re-challenged with docetaxel both as second- or thirdline chemotherapy.

Second-line chemotherapy for MHRPC is an unmet clinical need for a rapidly progressing and debilitating disease. There is no proven effective regimen in this setting and therefore patients who respond to first-line docetaxel chemotherapy should be considered for re-treatment.

This is the first evidence supporting docetaxel chemotherapy re-treatment using the three-weekly schedule in patients with MHRPC. This study clearly demonstrates that patients with MHRPC who initially respond to docetaxel chemotherapy maintain their sensitivity to subsequent retreatment. This could be explained by a hypothetical argument that a population of taxane-sensitive cells remained at the end of first-line chemotherapy, which maintained their intrinsic sensitivity and subsequently responded to chemotherapy retreatment. As none of the available chemotherapy drugs have shown significant benefit in this group, the case for docetaxel re-treatment grows stronger in MHRPC patients who initially respond to docetaxel and then progress after a period of biochemical remission. An alternative approach is to use intermittent docetaxel chemotherapy for patients with MHRPC, using up to six cycles of chemotherapy as part of first-line chemotherapy and reserving further cycles to be used on disease progression. However, prospective studies will be needed to validate this approach of intermittent three-weekly docetaxel chemotherapy.

In our database of docetaxel re-treatment, eight patients received $>12$ cycles of three-weekly docetaxel chemotherapy and interestingly one patient received 30 cycles (Fig. 3). Although, there is no recommended maximum cumulative dose for docetaxel, caution has to be exercised to avoid dose limiting toxicities. Perhaps, docetaxel in combination with one of the newer novel agents will show promise in the future.

\section{Acknowledgements}

The authors wish to thank Mrs. Sheena Collins for her excellent administrative support for the study.

\section{References}

1. Cancer stats monograph 2004: Cancer incidence, survival and mortality in the UK and EU. Cancer Research UK, 2004.

2. Taylor CD, Elson P and Trump DL: Importance of continued testicular suppression in hormone-refractory prostate cancer. J Clin Oncol 11: 2167-2172, 1993

3. Muthuramalingam SR, Patel K, Protheroe A: Management of patients with hormone refractory prostate cancer. Clin Oncol 16: 505-516, 2004.

4. Tannock IF, Osaba D, Stockler MR, et al: Chemotherapy with mitoxantrone plus prednisolone or prednisolone or prednisolone alone for symptomatic hormone resistant prostate cancer: a Canadian randomised trial with palliative end-point. J Clin Oncol 14: 1764-1765, 1996.

5. Tannock IF, de Wit R, Berry WR, et al: Docetaxel plus prednisolone or mitoxantrone plus prednisolone for advanced prostate cancer. N Engl J Med 351: 1502-1512, 2004.

6. Petrylak DP, Tangen CM, Hussain MH, et al: Docetaxel and Estramustine compared with mitoxantrone and prednisolone for advanced refractory prostate cancer. N Engl J Med 351: 1515-1527, 2004.

7. National Institute for Health and Clinical Excellence: Docetaxel for the treatment of hormone-refractory metastatic prostate cancer. NICE technology appraisal guidance 101, June 2006 (www.nice.org.uk/TA101)

8. Rosenberg JE, Weinberg VK, Kelly WK, et al: Activity of second-line chemotherapy in docetaxel-refractory hormonerefractory prostate cancer patients: randomized phase 2 study of ixabepilone or mitoxantrone and prednisone. Cancer 110: 556-563, 2007.

9. Nakabayashi M, Ling J, Xie W, et al: Response to vinorelbine with or without estramustine as second-line chemotherapy in patients with hormone-refractory prostate cancer. Cancer J 13: 125-129, 2007.

10. Ross RW, Beer TM, Jacobus S, et al: A phase 2 study of carboplatin plus docetaxel in men with metastatic hormonerefractory prostate cancer who are refractory to docetaxel. Cancer 112: $521-526,2008$ 
11. Bubley GJ, Carducci M, Dahut W, et al: eligibility and response guidelines for phase II clinical trials in androgen-independent prostate cancer: recommendations from the Prostate-Specific Antigen Working Group. J Clin Oncol 17: 3461-3467, 1999.

12. Armstrong AJ, Garrett-Mayer E, Ou Yang YC, et al: Prostatespecific antigen and pain surrogacy analysis in metastatic hormone-refractory prostate cancer. J Clin Oncol 25: 3965-3970, 2007.

13. Petrylak DP, Ankerst DP, Jiang CS, et al: Evaluation of prostate-specific antigen declines for surrogacy in patients treated on SWOG 99-16. J Natl Cancer Inst 98: 516-521, 2006.

14. Cara $S$ and Tannock IF: Retreatment of patients with the same chemotherapy: implications for clinical mechanisms of drug resistance. Ann Oncol 12: 23-27, 2001

15. Joshua AM, Nordman I, Venkataswaran R, et al: Weekly docetaxel as second line treatment after mitozantrone for androgen-independent prostate cancer. Intern Med J 35: 468-472, 2005.

16. Oh WK, Manola J, Babcic V, et al: Response to second-line chemotherapy in patients with hormone refractory prostate cancer receiving two sequences of mitoxantrone and taxanes. Urology 67: 1235-1240, 2006.
17. Michels J, Montemurro T, Murray N, et al: First- and second-line chemotherapy with docetaxel or mitoxantrone in patients with hormone-refractory prostate cancer: does sequence matter? Cancer 106: 1041-1046, 2006.

18. Calais da Silva FM, Calais Da Silva F, Bono A, et al: Phase III intermittent MAB versus continuous MAB. J Clin Oncol 24: 220s, 2006.

19. Tunn UW, Eckart O, Kienle EF, et al: Can intermittent androgen deprivation be an alternative to continuous androgen withdrawal in patients with PSA relapse? First results of the randomized prospective phase III clinical trial EC-507. J Urol 169 (Suppl 4): 396a, 2003.

20. Beer TM, Garzotto M, Henner WD, et al: Multiple cycles of intermittent chemotherapy in Metastatic androgen-independent prostate cancer. Br J Cancer 91: 1425-1427, 2004.

21. Beer TM, Ryan CW, Venner PM, et al: Intermittent chemotherapy in patients with metastatic androgen-independent prostate cancer: results from ASCENT, a double-blinded, randomized comparison of high-dose calcitriol plus docetaxel with placebo plus docetaxel. Cancer 112: 326-330, 2008. 